\title{
Pregnancy/abortion induced intracerebral hemorrhage during the first trimester in a young female
}

\author{
Nitesh Kumar ${ }^{1}$, Kapil Gupta ${ }^{2}$, Madhur Kalra ${ }^{3}$, Diwakar Verma ${ }^{1}$ \\ From ${ }^{1}$ Senior Resident, Department of Emergency Medicine, All India Institute of Medical Sciences (AIIMS), Basni, Jodhpur, Rajasthan, ${ }^{2}$ Consultant, \\ Department of Emergency Medicine, Manipal Hospital Delhi, Dwarka, Delhi, ${ }^{3}$ Consultant, Head, Department of Emergency Medicine, MM Hospital \\ Emergency Ward, MMU, Mullana, Haryana, India
}

\begin{abstract}
Intracranial hemorrhage is a relatively infrequent but devastating complication of pregnancy. Pregnancy-associated intracranial hemorrhage is a rare but severe complication. Mostly, the incidence of ICH occurs during the third trimester of pregnancy. In our case, an 18-year-old girl who had Glasgow coma scale score of 5/15, admitted to the hospital with no movement of left-sided upper and lower limb with right-sided facial deviation. Computed tomography (CT) brain revealed that the patient has right-sided temporoparietal bleed. The patient underwent right-sided hemicraniotomy. An obstetrical ultrasound report suggested a gestation of 12 weeks. The history of the patient highlighted an attempt of unsupervised termination of pregnancy using off-the-counter pills. In this case, we reported the rare intracerebral hemorrhage during the first trimester. Pregnancy has not been shown to be a direct cause of intracerebral bleed; hence, this case draws attention to the hazards of unsupervised termination of pregnancy.
\end{abstract}

Key words: First trimester, Intracranial hemorrhage, Pregnancy, Young female

I ntracranial hemorrhage (ICH) is a relatively infrequent but devastating complication of pregnancy that accounts for the third most common cause of non-obstetric related maternal mortality. Intracranial hemorrhage in pregnancy can manifest as extradural, subarachnoid, intracerebral, and intraventricular bleeds. Mostly, these events occurs during the second or $3^{\text {rd }}$ trimester $[1,2]$. The prevalence of stroke in pregnancy and puerperium appears in 10-34/100,000 parturient women and it is a major reason for the morbidity and mortality among pregnant women. In various countries, eclampsia and stroke during pregnancy are major factors of maternal and neonatal death [3]. Pregnancy-associated ICH is a rare but severe complication. The estimated mortality of pregnancy-associated ICH is $9-38 \%$, which contributes to more than $12 \%$ of all maternal mortality in most nations. The incidence of pregnancy-associated ICH in developing countries is even higher than that reported in other countries [4].

Importantly, ICH arising in pregnancy or postnatal is often due to new-onset preeclampsia and eclampsia, mainly when acute hypertension is uncontrolled [5]. Worldwide, $10 \%$ of all pregnancies become complicated due to hypertension,

\section{Access this article online}

Received - 05 March 2021

Initial Review - 20 March 2021

Accepted - 20 March 2021

DOI: $10.32677 /$ IJCR.2021.v07.i04.005 pre-eclampsia, and eclampsia. These factors are the major causes of maternal as well as antenatal morbidity and mortality. At the global level, it is evaluated that pregnancy-induced hypertension (PIH) affects about 5-8\% of all pregnant women [6]. Advanced maternal age, African American race, hypertensive diseases, coagulopathy, and tobacco abuse were all independent risk factors for pregnancy-related ICH [1]. In this backdrop, we present a firstof-its-kind case of intracerebral hemorrhage complicating the first trimester at 12 weeks of gestation without overt predisposing factors.

\section{CASE REPORT}

An 18-years-old unmarried female was brought to AIIMS, Jodhpur emergency department with a history of unconsciousness, leftsided weakness, and aphasia on June 11, 2019. The patient was shifted to the hospital after $1-2 \mathrm{~h}$ of unconsciousness. The patient's mother explained that the patient is not able to communicate with her along with no movement of the left-sided upper and lower limb with right-sided facial deviation. There was no history of fever, headache, nausea, vomiting, seizure, bowel, and bladder incontinence. The history of the patient revealed that there was no history of hypertension, diabetes, hospitalization, blood transfusion, and epilepsy. The patient has a normal menstrual

Correspondence to: Nitesh Kumar, Department of Emergency Medicine, AIIMS, Jodhpur, Rajasthan, India. E-mail: nitesh2007mmc@gmail.com

(C) 2021 Creative Commons Attribution-NonCommercial 4.0 International License (CC BY-NC-ND 4.0). 
history, menarche at 16 years of age with $5 / 28$, regular, moderate bleed. The last menstruation period was on June 2, 2019.

At the time of admission, the Glasgow Coma Scale (GCS) was 7/15. Pupils were bilateral equal $(3 \mathrm{~mm}$ ) and sluggishly reactive to light. The temperature was $98.8 \mathrm{~F}$, pulse rate was $64 / \mathrm{min}$, regular, blood pressure was 134/64 mmHg in both arms, and respiratory rate was $14 / \mathrm{min}$, abdomino-thoracic. On central nervous system (CNS) examination, the patient was having global aphasia, leftsided facial palsy, left-sided flaccidity, and poor power in the left side in both limbs at all joints. There were no signs of meningeal irritation and cerebellar signs. Cerebrovascular system (CVS) auscultation showed that S1 and S2 were heard normally in their respective area with no murmur and no carotid bruit. The abdomen was soft and non-tender.

Emergent computed tomography (CT) brain revealed rightsided gangliocapsular bleed (Fig. 1), however, the clinician sent her for an urgent magnetic resonance imaging (MRI) evaluation of the brain angiography. The report suggested no atrioventricular malformation.

The patient was started on intravenous mannitol, supportive medications, and shifted to a high dependency unit. The patient's general condition was better for the next 2 days. There was a sudden deterioration of neurological status and she developed anisocoria. The GCS became 5/15. Urgently, the patient was taken for right-sided decompression hemicraniotomy where her intubation was done. On the next day, anisocoria resolved and GCS was $7 / 15$, so, the patient was extubated and monitored for the next $24 \mathrm{~h}$. The patient had an ultrasound (USG) abdomen which was suggestive of 12-week gravid uterus. On June 15, 2019 , the patient was shifted to the neurosurgery ward. The same day, the patient developed respiratory distress and shifted to ICU where she was intubated for 3 days. On June 18, 2019, she was extubated and shifted to the neurosurgery ward the next day. Finally, the patient was survived and advised rehabilitation.

\section{DISCUSSION}

Pre-eclampsia is associated with maternal and fetal morbidity and mortality. In developing countries, the incidence of pre-eclampsia is 7 times higher as compared to the developed countries [7]. Cerebrovascular accidents during pregnancy are uncommon disorder but it is significant because case fatality is up to $20 \%$ [8]. It is difficult to measure the exact estimate of pregnancy-related vascular events. However, various studies have shown that stroke complicates almost 9-26 per 100,000 pregnancies and deliveries. Hemorrhage accounts for up to $38 \%$ of pregnancy-induced strokes.

Pregnancy-induced hypertension is a common obstetric complication that presents in $6 \%-8 \%$ of pregnancies [9]. In our case, we met an adolescent pregnant woman with spontaneous ICH. At the time of admission, GCS was 7/15. CNS examination showed global aphasia, left-sided facial palsy, left-sided flaccidity, and poor power in the left side in both limbs at all joints. Emergent non-contrast CT (NCCT) brain revealed

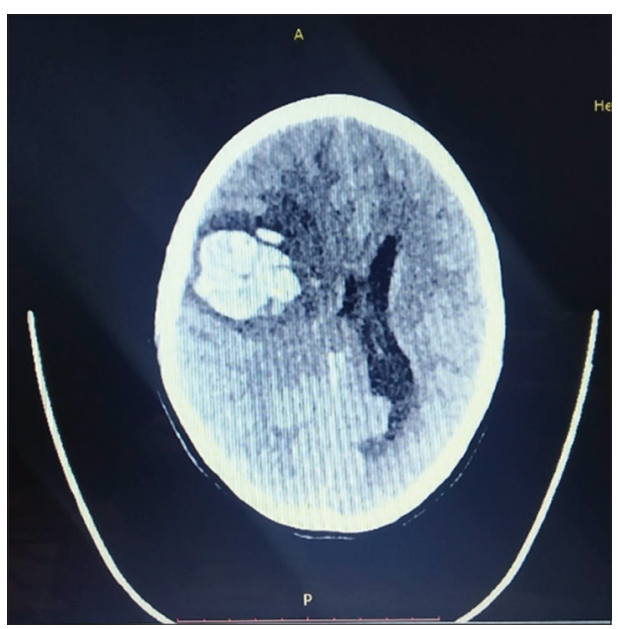

Figure 1: Computed tomography scan head showing intracranial hemorrhage

right-sided gangliocapsular bleeds. During pregnancy, increased cardiac output, plasma volume, and changes in the vessel wall increase the risk of rupture of small aneurysms or arteriovenous malformations [10]. Suspicious neurological signs and symptoms in a pregnant patient should be thoroughly evaluated. The mean gestational age at the time of stroke observed is 32 weeks and 6 weeks postpartum [8].

However, in our case, ICH developed in the first trimester of pregnancy was retrospectively sought after and was confirmed that there was no evidence of $\mathrm{AV}$ malformations/pre-eclampsia/ coagulopathy. Verma and Agrawal have presented a case study of a woman who had developed spontaneous ICH during the pregnancy. The patient has altered sensorium, aphasia, and hemiparesis. The MRI findings revealed intracerebral hemorrhage [8].

The mechanism for the development of ICH in our case is not easily explained; however, a high index of suspicion should be maintained throughout the period of pregnancy and the postpartum period. Furthermore, women in child-bearing age group with unexplained vascular events should be screened for pregnancy. Matsuda et al. communicated that $\mathrm{ICH}$ is a rare complication during gestation. A 33-year-old female at 20 weeks of gestation suffered from hematoma due to ICH [11].

Management of the intracerebral hemorrhage follows standard neurosurgical principles. Surgical evacuation of a clot is indicated if there is a declining conscious state or worsening neurological deficit. Of course, surgery remains the appropriate treatment when there is a progression of the clinical state to one of deteriorating consciousness or progressive neurological deficit. This case highlighted that ICH may occur during the first trimester so, early diagnosis and treatment of ICH is necessary during pregnancy to prevent a serious complication such as ICH.

\section{CONCLUSION}

Intra-cerebral bleed is one of the major causes of maternal morbidity and mortality among pregnant women. The incidence of this catastrophic condition is variable; it has specific confront in diagnosis and management. Pregnancy has not been shown 
to be a direct cause of intracerebral bleed; hence, this case draws attention to the hazards of unsupervised termination of pregnancy. More evidence are required to develop guidelines for prevention and management during pregnancy since there is a scarcity of data available regarding the use of over-the-counter drugs for unsupervised termination of pregnancy. An integrative approach involving obstetricians, neurologists, and intensivists are important in the management of ICH during pregnancy.

\section{REFERENCES}

1. Fairhall JM, Stoodley MA. Intracranial haemorrhage in pregnancy. Obstet Med 2009;2:142-8.

2. Ghaly RF, Candido KD, Sauer R, Knezevic NN. Complete recovery after antepartum massive intracerebral hemorrhage in an atypical case of sudden eclampsia. Surg Neurol Int 2012;3:65.

3. Malgorzata W, Stachowiak I, Nowak B, Wiszniewski P. Haemorrhagic stroke during pregnancy may be successful a case study of two cases. Obstet Gynecol Int J 2015;2:109-11.

4. Liang ZW, Lin L, Gao WL, Feng LM. A clinical characteristic analysis of pregnancy-associated intracranial haemorrhage in China. Sci Rep 2015;5:9509.
5. Aoyama K, Joel R. Pregnancy and risk of intracerebral hemorrhage. JAMA Netw Open 2020;3:e202844.

6. Yadav P, Mukherjee A, Kumar R. Spontaneous subdural hematoma in a multigravida with chronic kidney disease and superadded pre-eclampsia detected by MRI: A case report. Int J Res Med Sci 2019;7:1381-3.

7. Pareek S, Ramawat Y, Kumar N, Lal B, Kumar V. Pre-eclampsia: A hypertensive disorder during gestation. J Nurse Midwifery Matern Health 2020;6:19-22.

8. Verma K, Agrawal S. A life threatening intracerebral hemorrhage during pregnancy. Int J Reprod Contracept Obstet Gynecol 2015;4:840-2.

9. Luk A, Ma R, Lam C, Tam W, Lo A, Ng E, et al. A 21-year-old pregnant woman with hypertension and proteinuria. PLoS Med 2009;6:e1000037.

10. Treadwell S, Thanvi B, Robinson T. Stroke in pregnancy and the puerperium. Postgr Med J 2008;84:238-45.

11. Matsuda M, Yamazaki M, Osada J, Hirata M, Amaya F. A case of emergency cesarean section and craniotomy in a 26 week pregnant woman. Masui 2015;64:192-5.

Funding: None; Conflicts of Interest: None Stated.

How to cite this article: Kumar N, Gupta K, Kalra M, Verma D. Pregnancy/abortion induced intracerebral hemorrhage during the first trimester in a young female. Indian J Case Reports. 2021;7(4):140-142. 